\title{
Cytomorphological features of metastatic squamous cell carcinoma in serous effusions
}

\author{
C. C. Huang* and C. W. Michael ${ }^{\dagger}$ \\ *Department of Pathology, University of Michigan, Ann Arbor, MI, USA and ${ }^{\dagger}$ Department of Pathology, Case Western \\ Reserve University/University Hospitals, Case Medical Centre, Cleveland, OH, USA
}

Accepted for publication 10 March 2013

C. C. Huang and C. W. Michael

Cytomorphological features of metastatic squamous cell carcinoma in serous effusions

Objective: To study the cytomorphological features of squamous cell carcinoma (SCC) in serous effusions. Methods: Conventional smears from 23 SCC were reviewed for the following features: cellularity, single cells, cell clusters, long cords, squamous pearls, cell-in-cell, 'windows', polygonal cells, anucleated cells, tadpole cells, fibre cells, third-type cells, refractile cytoplasmic ring, two-tone cytoplasm, cytoplasmic density, cytoplasmic vacuoles, signet ring cells, nuclear/cytoplasmic ratio, nuclear hyperchromasia, irregular nuclear membrane, nuclear centricity, double or multiple nuclei, nucleolus, anaplastic cells and mitosis. Subsequently, we also reviewed 15 reactive mesothelium, 18 mesotheliomas and 24 adenocarcinomas for comparison.

Results: Most SCC smears comprised mixed populations of single cells and cell clusters. Keratinized squamous cells and squamous pearls were diagnostic for well-differentiated SCC. Squamous eddies, appreciated on the cell block sections appearing as two-dimensional cell clusters with a swirling pattern, were characteristic for SCC. Poorly differentiated/third-type cells with atypical cytoplasmic keratinization, manifest as twotone cytoplasm with a well-defined border and occasionally a refractile central ring, were an important clue for the squamous origin of the cells. Features including cell-in-cell, cytoplasmic vacuolation, double or multinucleated cells, anaplastic cells and mitotic figures were present in various degrees, and were not of diagnostic value.

Conclusions: Certain cytological features are diagnostic for SCC, whereas other features of poorly differentiated SCC often overlap with those of reactive mesothelial cells, mesothelioma, metastatic adenocarcinoma or urothelial carcinoma in serous effusions. The combination of clinical history, appropriate immunohistochemical panel and cytomorphological features are important to ensure a correct diagnosis.

Keywords: squamous cell carcinoma, serous effusions, metastatic carcinoma, cytopathology, differential diagnosis

\section{Introduction}

Malignant effusions are commonly a manifestation of disseminated malignancy and indicate the end stage of disease. Squamous cell carcinoma (SCC) is

Correspondence:

C. W. Michael, Professor of Pathology, Department of Pathology, Case Western Reserve University/University Hospitals Case Medical Center, 11100 Euclid Ave Rm 212B, Cleveland, OH 44106, USA

Tel.: +1 216844 0137; Fax: +1 216844 1810;

E-mail: claire.michael@case.edu one of the most common cancers and is a leading cause of cancer-related death. However, serous effusions caused by SCC are extremely rare. Review of the literature shows a few recent case reports on the cytomorphology of metastatic SCC to serous cavities. ${ }^{1-4}$ The largest series of SCC in serous effusions to date was published in 1989, in which a total of 46 patients with metastatic SCC to pleural, peritoneal or pericardial cavities were identified in 9297 serous effusions during a period of 33 years. ${ }^{5}$

In this study, we evaluated the cytomorphological features of SCC in 23 serous effusions to identify the 
diagnostic features and potential pitfalls in these cases.

\section{Methods}

A total of 36 cases of metastatic SCC to pleural, peritoneal or pericardial fluids were retrieved from the pathology database at the University of Michigan between 1989 and 2011 when a total of 18626 serous fluids were accessioned. While it would have been interesting to evaluate the incidence of malignant effusions in patients with a known history of SCC and presenting with effusion, this was not possible as our laboratory information system does not store the clinical information provided on the initial requisition.

Based on regulatory guidelines and recommendations by the College of American Pathologists, slides and paraffin blocks of effusions can be discarded after 10 years. Consequently, of those 36 cases, smears were available for review in 23 cases comprising 17 pleural fluids, five peritoneal fluids and one pericardial fluid. All relevant clinical information was obtained from the patients' medical records. For each case, two air-dried Diff-Quik ${ }^{\circledR}$-stained smears, two alcohol-fixed Papanicolaou-stained smears and a cell block were prepared. In bloody or low-cellularity samples, a ThinPrep ${ }^{\circledR}$ was also prepared. The smears were reviewed and evaluated for the presence of the following features: cellularity, single cells, cell clusters, squamous pearls, long cords, cell-in-cell, 'windows' between the cells, polygonal cells, anucleated cells, tadpole cells, fibre cells, third-type cells, refractile cytoplasmic ring, two-tone cytoplasm, cytoplasmic density, cytoplasmic vacuoles, signet ring cells, nuclear/cytoplasmic (N/C) ratio, nuclear hyperchromasia, irregular nuclear membrane, nuclear centricity, nucleolus, double nuclei, multiple nuclei, anaplastic cells and mitosis. All cases had a well-documented history of SCC confirmed by resection of the primary tumour and poorly differentiated cases were confirmed by immunohistochemical staining performed on cell block sections as previously described. ${ }^{\mathbf{6}, 7}$ A semi-quantitative method was used to quantitate each of the cytomorphological features. In general, a feature is considered as high $(3+)$ when easily detected at $10 \times$ magnification, moderate $(2+)$ when detected at $20 \times$ or low $(1+)$ when only detected at $40 \times$ magnification.

After our review of the SCC cases, we also reviewed smears from 15 with reactive mesothelium
(RM), 18 malignant mesotheliomas (MM) and 24 adenocarcinomas (ADC : six Müllerian, two pancreas, seven breast, seven lung and two colorectal in origin) for the same features as a control group.

\section{Results}

The 36 patients included in this study were 15 males and 21 females with average age of 57 years. The malignant effusions consisted of 30 pleural fluids $(83 \%)$, five peritoneal fluids (14\%) and one pericardial fluid $(3 \%)$. The effusions represented metastases from various primary sites of SCC (Table 1). The lung was the most common primary site for all the malignant effusions. Primary lung SCC (15 cases) accounted for $42 \%$ of all the metastases and $50 \%$ of the pleural metastases. Cervical SCC (four cases) was the most common primary for peritoneal serous effusions and accounted for $80 \%$ of the peritoneal metastases.

Smears were available for review in 23 cases comprising 17 pleural, five peritoneal and one pericardial effusion. The cytomorphological features of the SCC together with those of RM, MM and ADC in serous effusions are summarized in Table 2. The cellularity varied considerably among the smears. The spectrum of cases in this study ranged from well to poorly differentiated SCC. Poorly differentiated cells, using the term 'third-type cells' coined by Ruth Graham, ${ }^{8}$ are defined as round or oval cells with a high N/C ratio, dense cytoplasm, distinct cell borders and large nuclei with irregular nuclear membranes and hyperchromatic clumpy chromatin on Papanicolaoustained smears (Figure la), were present in all the

Table 1. Primary sites of metastatic squamous cell carcinoma in serous effusions

\begin{tabular}{llll}
\hline Cavity & Primary sites & Total cases & Reviewed cases \\
\hline Pleural & Lung & 15 & 11 \\
& Tongue & 4 & 2 \\
& Larynx & 3 & 1 \\
& Oesophagus & 2 & 2 \\
& Mandible & 2 & 0 \\
& Renal pelvis & 1 & 0 \\
& Palate & 1 & 0 \\
& Parotid gland & 1 & 1 \\
\multirow{5}{*}{ Peritoneal } & Tonsil & 1 & 0 \\
\multirow{5}{*}{ Pericardial } & Cervix & 4 & 4 \\
& Larynx & 1 & 1 \\
& Parotid gland & 1 & 1 \\
\hline
\end{tabular}




\begin{tabular}{|c|c|c|c|c|}
\hline & $\operatorname{SCC} n(\%)$ & $\mathrm{RM} n(\%)$ & $\operatorname{MM} n(\%)$ & $\operatorname{ADC} n(\%)$ \\
\hline $\begin{array}{l}\text { Total smears reviewed } \\
\text { Smear patterns }\end{array}$ & 23 & 15 & 18 & 24 \\
\hline High cellularity & $5(22)$ & $13(87)$ & $18(100)$ & $14(58)$ \\
\hline Single cell & $23(100)$ & $15(100)$ & $18(100)$ & $1(4)$ \\
\hline Cell Cluster & $15(65)$ & $11(73)$ & $18(100)$ & $23(96)$ \\
\hline Squamous pearl & $3(13)$ & $0(0)$ & $0(0)$ & $0(0)$ \\
\hline Long cord & $2(9)$ & $0(0)$ & $0(0)$ & $0(0)$ \\
\hline Cell in cell & $4(17)$ & $10(67)$ & $13(72)$ & $3(13)$ \\
\hline Cell windows & $8(35)$ & $15(100)$ & $18(100)$ & $0(0)$ \\
\hline \multicolumn{5}{|l|}{ Cytological features } \\
\hline Polygonal cell & $7(30)$ & $0(0)$ & $0(0)$ & $0(0)$ \\
\hline Anucleated cell & $2(9)$ & $0(0)$ & $0(0)$ & $0(0)$ \\
\hline Tadpole cell & $4(17)$ & $0(0)$ & $0(0)$ & $0(0)$ \\
\hline Fibre cell & $5(22)$ & $0(0)$ & $0(0)$ & $0(0)$ \\
\hline Third-type cell & $23(100)$ & $0(0)$ & $0(0)$ & $0(0)$ \\
\hline Refractile cytoplasmic ring & $13(57)$ & $0(0)$ & $0(0)$ & $0(0)$ \\
\hline Two-tone cytoplasm & $9(39)$ & $15(100)$ & $18(100)$ & $7(29)$ \\
\hline Dense cytoplasm & $23(100)$ & $8(53)$ & $8(44)$ & $0(0)$ \\
\hline Cytoplasm vacuoles & $12(52)$ & $9(60)$ & $5(28)$ & $19(79)$ \\
\hline Signet ring cells & $3(13)$ & $0(0)$ & $0(0)$ & $5(20)$ \\
\hline High N/C ratio & $23(100)$ & $0(0)$ & $0(0)$ & $24(100)$ \\
\hline Nuclear hyperchromasia & $23(100)$ & $0(0)$ & $1(6)$ & $24(100)$ \\
\hline $\begin{array}{l}\text { Irregular nuclear } \\
\text { membrane }\end{array}$ & $21(91)$ & $0(0)$ & $0(0)$ & $22(91)$ \\
\hline Nuclei centricity & $23(100)$ & $15(100)$ & $18(100)$ & l (4) \\
\hline Nucleoli & $15(65)$ & $15(100)$ & $18(100)$ & $19(79)$ \\
\hline Double nuclei & $4(17)$ & $2(13)$ & $5(28)$ & $1(4)$ \\
\hline Multiple nuclei & $3(13)$ & $0(0)$ & $0(0)$ & $0(0)$ \\
\hline Anaplastic cells & $2(9)$ & $0(0)$ & $0(0)$ & $7(29)$ \\
\hline Mitosis & $1(4)$ & $1(7)$ & $2(11)$ & $15(68)$ \\
\hline
\end{tabular}

Table 2. Cytological feature comparison among squamous cell carcinoma, reactive mesothelium, malignant mesothelioma and adenocarcinoma in serous effusions

SCC, squamous cell carcinoma; RM, reactive mesothelium; MM, malignant mesothelioma; ADC, adenocarcinoma.

squamous carcinoma specimens and were absent in RM, MM, and adenocarcinomas. Atypical keratinization of third-type cells as previously described by John K. Frost, ${ }^{9}$ characterized by sharp demarcation of the ecto-endoplasmic border as a result of hyalinization of the ectoplasm and normal texture of the endoplasm, refractile ring formation, and a crisply thin well-defined cell border, were identified in more than half of the cases (Figure lb) but none of the controls. Polygonal cells were defined as flat cells with central dense hyperchromatic chromatin. Tadpole cells were elongated cells with a bulbous end containing a nucleus and a long tail of stretched cytoplasm. Fibre cells or spindle cells were slender with centralized elongated nuclei (Figure 2). Keratinized polygonal cells, anucleated cells, tadpole cells and fibre cells were present in seven, two, four and five of 23 cases, respectively, and were not seen in controls. These keratinized cells often coexisted. Keratinized cells had a bright orangeophilic cytoplasm on Papanicolaou staining and bright robin blue on Diff-Quik staining. Cells with variable cytoplasmic density were seen in all 23 cases. Two-tone cytoplasm was detected in nine cases (Figure 3, inset). It appeared as cyanophilic ectoplasm and eosinophilic/ amphophilic endoplasm and a well-defined border by Papanicolaou staining, or as light blue perinuclear cytoplasm and peripheral dark blue cytoplasm and a well-defined border by Diff-Quik staining.

Cytoplasmic vacuolation was identified in 12 cases, mostly appearing as scattered dot-like vacuoles (Figure 3), but were also seen in some cases of RM, $\mathrm{MM}$ and ADC. Abundant large disfiguring cytoplasmic vacuolation (Figure 4, inset) and signet ring 

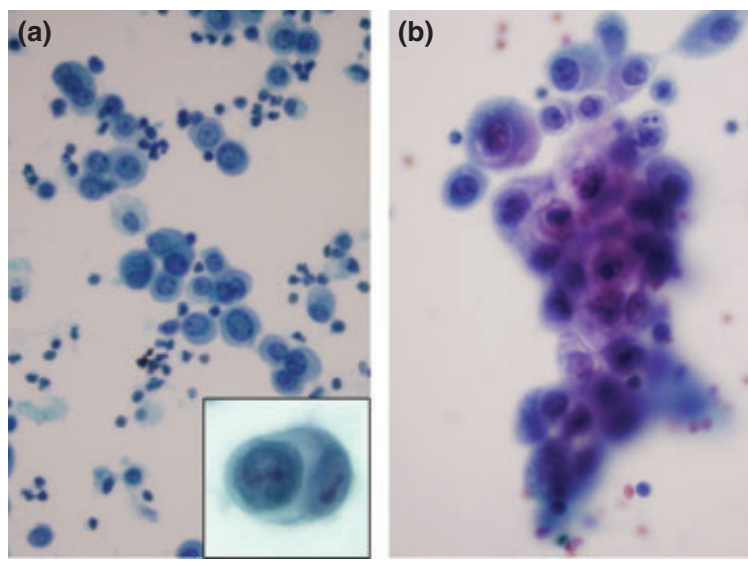

Figure 1. (a) Moderately differentiated third-type squamous cell carcinoma cells with dense cytoplasm, welldefined cell borders, round or oval nuclei and inconspicuous nucleoli; cell-in-cell is occasionally seen mimicking cellular clasping (inset). (b) Refractile rings of atypical keratinization formed by demarcation of hyalinized ectoplasm and remaining textured endoplasm. Papanicolaou $\times 600$.

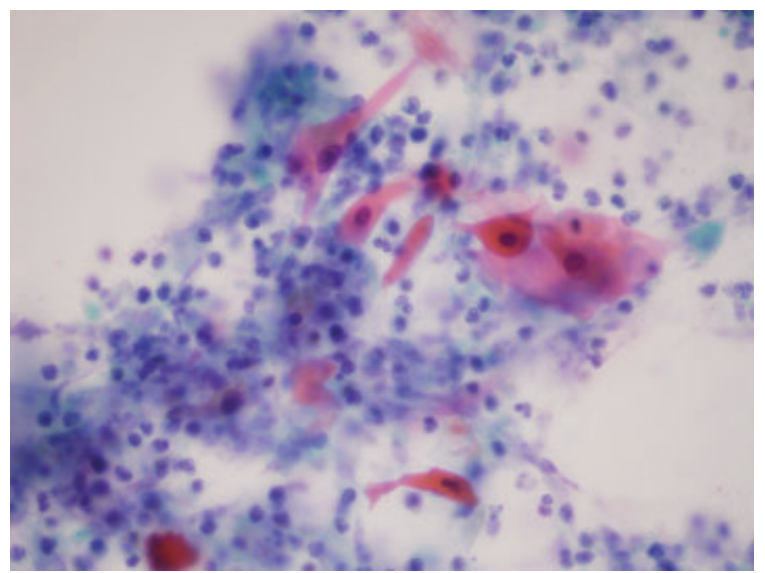

Figure 2. Polygonal cells, tadpole cells, fibre cells and anucleated cells are commonly identified in keratinized welldifferentiated squamous cell carcinoma. Papanicolaou $\times 600$.

cells (Figure 3) were occasionally seen. Double or multiple nucleated cells (Figure la), anaplastic cells (Figures 3 and 5b) and mitotic were not common and were not specific for SCC.

Cell clusters, formed by aggregation of third-type cells, were present in 15 of 23 cases and were also seen in controls. In SCC these clusters were usually large, two-dimensional with irregular or a geographic-like outline (Figures 4, 5 and 6). Loose cell

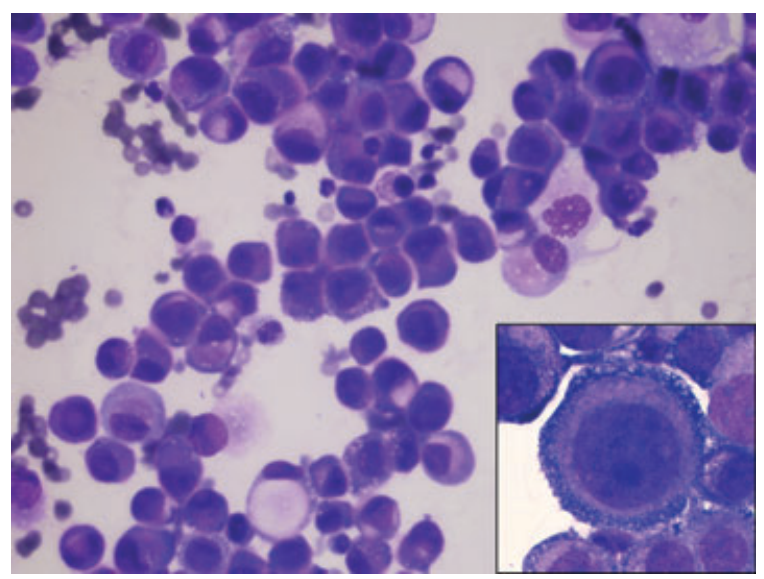

Figure 3. Third-type cells form long cords with straight linear empty spaces between the cells. Scattered dot-like cytoplasmic vacuolations are frequent with occasionally signet ring cells and anaplastic cells (Diff-Quik $\times 600$ ); twotone cytoplasm is evident in some cells (inset $\times 1000$ ).

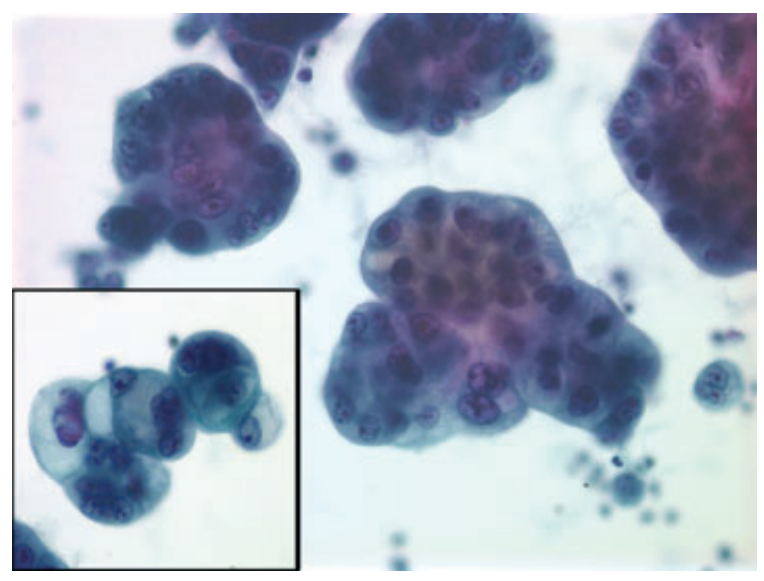

Figure 4. Cell clusters are two-dimensional with smooth borders in this poorly differentiated squamous cell carcinoma. Small groups of cell with disfiguring cytoplasmic vacuolation are occasionally present (inset): Papanicolaou $\times 600$.

clusters were commonly formed by moderately differentiated third-type cells (Figure 5a); whereas tight cell clusters were often formed by either moderately (Figure 6) or poorly differentiated SCC cells (Figure 4). Squamous eddies, formed by swirling of malignant cells with elongated, flatted cells at the periphery of cell clusters were commonly seen in cellular specimens of moderately differentiated SCC (Figure 6) and were best appreciated in cell block sections (Figure 7). Squamous pearls, formed by a 

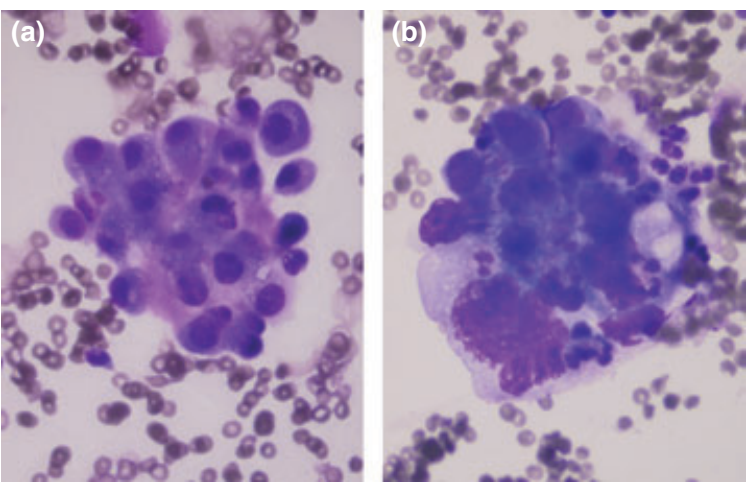

Figure 5. (a) A loose two-dimensional cell cluster formed by moderately differentiated squamous cell carcinoma (SCC). (b) A tight cell cluster is often seen in poorly differentiated SCC; an anaplastic cell and cytoplasmic-vacuolated cells are also presented. Diff-Quik $\times 600$.
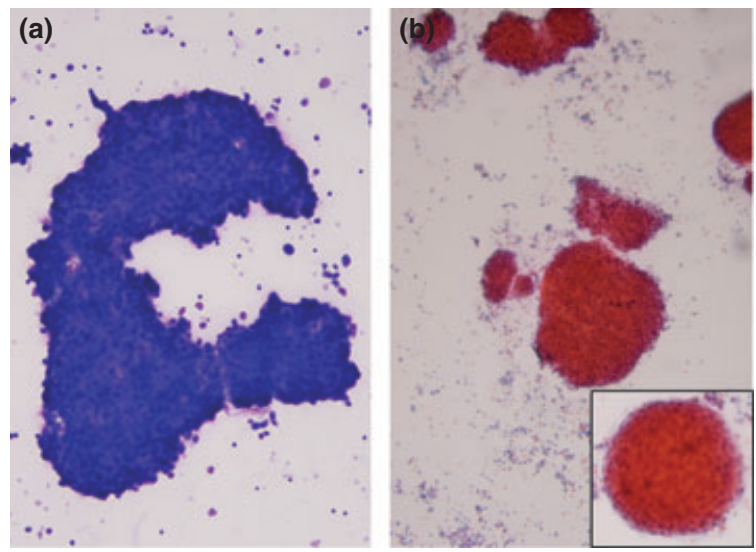

Figure 6. (a) Cell clusters are variable in size and shape with irregular rigid borders in this moderately differentiated squamous cell carcinoma (Diff-Quik $\times 200$ ). (b) The clusters are wrapped by elongated and flattened cells (Papanicolaou $\times 200$, inset $\times 600$ ).

group of cells tightly wrapped around each other, were identified in three cases (Figure 8). Long cords, defined as a row of third-type cells, were identified in two cases (Figure 3). Cell-in-cell (Figure la, inset) and cell windows (Figure 3) were seen in four and eight of the cases, respectively. In those cases, the intercellular windows appeared more as a tight straight space rather than the biconvex spaces traditionally seen between mesothelial cells. Our review of Diff-Quik-stained smears revealed that these third-type cells exhibited strikingly similar features

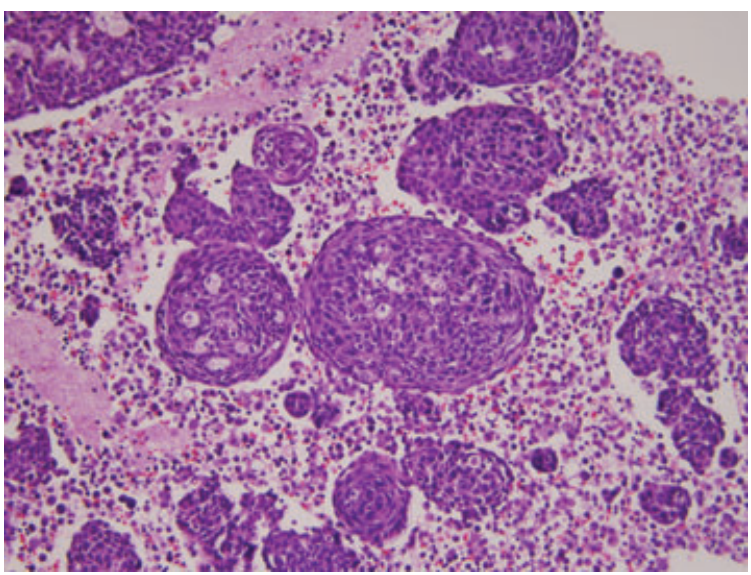

Figure 7. Squamous eddies, formed by swirling of moderately differentiated squamous cell carcinoma within the cell clusters are characteristic on cell block sections. Haematoxylin and eosin $\times 400$.

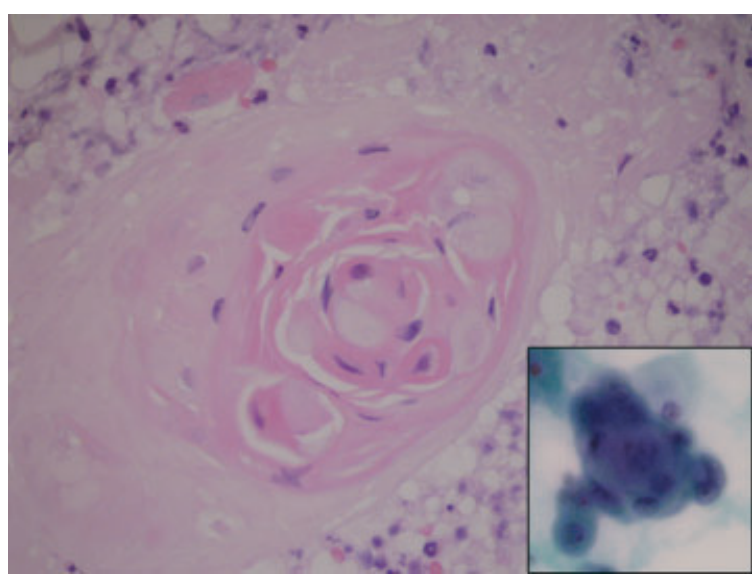

Figure 8. Squamous pearl, formed by groups of cells tightly wrapped around each other identified on the cell block section (haematoxylin and eosin $\times 400$ ) and smear (inset, Papanicolaou $\times 600$ ).

to those previously described on Papanicolaoustained smears (Figure 5). Interestingly, some features such as two-tone cytoplasm and well-defined cell borders were easier to detect on Diff-Quikstained smears underscoring its significance as a complementary stain in these cases.

Various panels of immunohistochemical staining were performed on cell block sections as previously published. ${ }^{6,7}$ Table 3 summarizes the reaction of our cases to various antibodies performed over the years. Since 2006, we confirm the diagnosis with a positive P63 or CK5/6 and negative WT-1. Table 4 summarizes a comparison of the immunoreaction of the 
Table 3. Immunostaining reactivity in squamous cell carcinoma metastases in serous effusions

\begin{tabular}{|c|c|c|c|c|c|c|c|c|c|c|}
\hline Case, effusion, primary & Cam 5.2 & K903 & $\mathrm{CK} 5 / 6$ & BerEP4 & MOC-31 & CEA & Calretinin & Mesothelin & WT1 & p63 \\
\hline 1. Pl Lung & + & + & + & - & + & - & - & + & - & + \\
\hline 2. Pl Oesophagus & + & + & + & - & - & ND & ND & - & - & + \\
\hline 3. Pl Lung & - & + & + & - & + & - & - & - & - & + \\
\hline 4. Pl Tongue & + & + & + & - & + & - & - & - & - & + \\
\hline 5. Pl Larynx & ND & + & + & - & - & ND & - & - & - & + \\
\hline 6. Pl Lung & + & + & + & - & + & ND & - & + & - & + \\
\hline 7. Pl Lung & + & + & + & - & + & + & - & - & - & + \\
\hline 8. Pc parotid & - & + & + & - & + & + & - & + & - & + \\
\hline 9. Pl Lung & + & + & + & - & + & - & - & + & - & + \\
\hline 10. Pl Lung & - & + & + & - & + & - & - & + & - & - \\
\hline 11. Pl Tongue & + & + & + & - & - & + & - & - & - & + \\
\hline 12. Pl Lung & + & + & + & - & - & ND & - & + & - & - \\
\hline 13. Pe Cervix & + & + & + & - & - & - & - & + & - & + \\
\hline 14. Pl Lung & - & + & + & - & + & ND & - & + & - & - \\
\hline 15. Pl Oesophagus & ND & + & + & - & - & - & - & + & - & + \\
\hline Positive/total & $9 / 13$ & $15 / 15$ & $15 / 15$ & $0 / 15$ & $9 / 15$ & $3 / 10$ & $0 / 14$ & $9 / 15$ & $0 / 15$ & $12 / 15$ \\
\hline
\end{tabular}

Pl, pleural effusion; Pc, pericardial effusion; Pe, peritoneal effusion; ND, not done or not available for review.

Table 4. Immunostaining reactivity of squamous cell carcinoma (SCC) and its differential diagnoses with the antibodies used in Table 3

\begin{tabular}{|c|c|c|c|c|}
\hline Antibody & $\begin{array}{l}\text { SCC * } \\
n(\%)\end{array}$ & $\begin{array}{l}\mathrm{RM}^{*} \\
n(\%)\end{array}$ & $\begin{array}{l}\text { MM * } \\
n(\%)\end{array}$ & $\begin{array}{l}\mathrm{ADC}{ }^{*} \\
n(\%)\end{array}$ \\
\hline P63 & $12 / 15(80)$ & $0 / 15(0)$ & $0 / 18(0)$ & $3 / 24(13)$ \\
\hline CK5/6 & $15 / 15(100)$ & $15 / 15(100)$ & $17 / 18(94)$ & $11 / 24(46)$ \\
\hline K903 & $15 / 15(100)$ & $11 / 14(79)$ & $11 / 12(92)$ & $13 / 24(93)$ \\
\hline Cam 5.2 & $9 / 13(69)$ & $14 / 15(93)$ & $12 / 12(100)$ & $12 / 12(100)$ \\
\hline Calretinin & $0 / 9(0)$ & $15 / 15(100)$ & $18 / 18(100)$ & $0 / 12(0)$ \\
\hline WT1 & $0 / 15(0)$ & $15 / 15(100)$ & $18 / 18(100)$ & $0 / 24(0)$ \\
\hline Moc31 & $9 / 15(60)$ & 4/15 (27) & 6/17 (35) & $10 / 10(100)$ \\
\hline Mesothelin & $9 / 15(60)$ & $2 / 4(50)$ & $8 / 17$ (47) & $10 / 10(100)$ \\
\hline BerEP4 & $0 / 15(100)$ & $0 / 15(0)$ & $1 / 18(6)$ & $10 / 12(83)$ \\
\hline CEA & $3 / 10(30)$ & $0 / 15(0)$ & $0 / 18(0)$ & $10 / 12(83)$ \\
\hline
\end{tabular}

RM, reactive mesothelium; MM, malignant mesothelioma; ADC, adenocarcinoma.

*Positive cases/total number stained and available for review.

SCC and its differential diagnoses to commonly used antibodies.

\section{Discussion}

Malignancies of lung, breast, ovary and lymphoma are the most common causes of malignant effusions, and they constitute more than $75 \%$ of malignant pleural effusions. ${ }^{10}$ The direct or indirect spread of breast, ovarian, endometrial, gastric, colonic and pancreatic carcinomas contribute up to $80 \%$ of malignant peritoneal effusions. ${ }^{11}$ While SCC is a common malignancy, it rarely contributes to malignant effusions. Only 36 cases were identified in 18626 cases of serous fluids in our pathology database over a period of 22 years. SCC of the lung and of head and neck region were the main primary sources of metastasis to the pleural and pericardial cavities whereas cervical SCC was the most common source of metastasis to the peritoneal cavities. Our results are consistent with previous studies. ${ }^{2-4}$

The diagnosis of SCC in serous effusions is not difficult in most cases when the patient's history is known and the characteristic cytomorphological 
features are present, particularly the presence of keratinized cells and squamous eddies. However, the diagnosis can be challenging and easily misclassified when SCC is less differentiated. The diagnosis of metastatic moderately or poorly differentiated SCC to serous effusions poses a diagnostic challenge owing to its overlapping features with other pathological effusions. The differential diagnoses of SCC in serous effusions include MM, ADC and rarely metastatic urothelial carcinoma (UC) or even reactive mesothelium. The presentation of cellular smears with both cellular clusters and single cells showing two-tone cytoplasm and intercellular windows mimics that of MM. However, in MM, the cell clusters are three-dimensional with scalloped berry-like borders. Other characteristic features of mesothelial origin such as the sub-membranous glycogen vacuoles, the vague cytoplasmic borders attributed to the circumferential microvilli, and the characteristic cellular clasping with pinched cytoplasm of adjacent cells are readily detected. Also in MM, the background single cells are widely variable in size ranging from that of a normal mesothelial cell to a gigantic size approaching that of the small adjacent morules, and multinucleation is usually conspicuous. Cytological atypia is subtle in $\mathrm{MM}$ with low N/C ratio, relatively smooth nuclear contours and less nuclear hyperchromasia. ${ }^{12-15}$ In contrast, cell clusters of poorly differentiated SCC are larger and more variable in size throughout the smear and exhibit irregular borders. SCC cells are relatively uniform in size and have conspicuous cytological atypia. Other features of squamous differentiation as described above serve as clues that can alert the reviewer to the possibility of SCC origin. RM can exhibit significant nuclear atypia with nuclear enlargement, coarse chromatin and an irregular nuclear contour or prominent nucleoli. However, the cytological atypia is never as striking as that of SCC and features characteristic of mesothelial origin, as described above, can easily be recognized. It is noteworthy that in rare cases of SCC cytoplasmic glycogen droplets can also be detected; however, in those cases the droplets are usually larger and located in the cytoplasm rather than the sub-membranous position in mesothelial cells. Metastatic adenocarcinoma is the most common misclassification for poorly differentiated SCC as both adenocarcinoma and SCC present with cell clusters and high nuclear atypia. This pitfall is compounded with the fact that SCC will positively react to most traditional immunizations used to confirm adenocarcinoma such as CEA, MOC-31, BerEP4 etc. $^{6,7}$ While the literature reports high immunoreactivity to BerEp4 in SCC, our cases were all non reactive. This difference could perhaps be attributed to the difference of antigen expression in the exfoliated cells we tested versus the tissue samples that were tested in the reported literature. Cell clusters of adenocarcinoma are usually three-dimensional with a common or smooth circumference. The presences of acinar structures, large cytoplasmic vacuoles or signet ring cells are suggestive of the glandular origin. However, it is important to recognize that these features are not specific for adenocarcinoma, and can be detected in RM, MM, SCC and other malignancies. ${ }^{14}$ In general, the presence of cytoplasmic vacuoles and signet ring cells is far more common in ADC than in those of SCC. In ADC, cell clusters are three-dimensional with a syncytial appearance of the malignant cells within the clusters. In contrast, the cell clusters in SCC are two-dimensional, and the cells usually have distinct cell borders within the cluster.

Metastatic UC to serous effusions is extremely rare $\mathrm{e}^{16-18}$ and presents as single cells with or without cell clusters. The single cells commonly present with a finely vacuolated cytoplasm, enlarged nuclei with an increased N/C ratio, irregular nuclear membrane, and hyperchromatic coarse chromatin and frequent prominent nucleoli. The cell clusters are usually small and two-dimensional. Many cytomorphological features of UC overlap with those of SCC. However, UC lacks characteristic features of squamous differentiation. 'Cell wrapping', a key feature in UC and defined as tumour cells partially or completely wrapped by one or more tumour cells with the nuclei molded in a crescent form around the cytoplasm of the central cell, are not seen in SCC. ${ }^{19}$

Another challenge is when SCC co-exists with other primary malignancies such as ADC or UC. However, co-existence with other malignancies is rare. ${ }^{5}$ In our study, only four patients presented with second primary malignancy including oesophageal adenocarcinoma, colon adenocarcinoma, chronic lymphocytic lymphoma and basal cell carcinoma (one case of each). Nevertheless, immunohistochemical stains are commonly used to distinguish such differential diagnoses in some difficult cases. Our present results are consistent with previous studies and show that a panel of K903, CK5/6, p63, calretinin, WT-1 and Ber-EP4 has a very high sensitivity and specificity in the differentiation of SCC 
from mesothelial cells or ADC. ${ }^{6,7}$ It is important to recognize that some of these markers may cross react with MM, SCC or ADC. ${ }^{7,20}$ Therefore, a focused immunohistochemical panel should always be used and interpreted judiciously. In pleural effusions, the main differential diagnosis includes ADC of the lung and MM, whereas in peritoneal effusions, the main differential diagnosis includes gastric or ovarian ADC. In these settings, p63 and WT-1 are especially valuable in the differentiated diagnosis. p63, a strong nuclear stain expressed in $80-100 \%$ of SCC but rarely expressed in lung ADC or MM, is a very useful confirmatory positive marker for SCC. WT-1 is a positive marker for MM, ovarian or a Mullerian tumour, and is a negative marker for SCC and lung ADC.

In conclusion, metastatic SCC is very rare in serous effusions. The diagnosis of metastatic SCC in effusions is usually not difficult with a clinical history and characteristic cytomorphology. However, certain cytological features overlap with those of RM, MM, metastatic ADC or UC. The combination of clinical history, appropriate immunohistochemical panel and cytomorphological features are important to ensure a correct diagnosis.

\section{References}

1. Nieto-Llanos S, Vera-Roman JM. Squamous cell carcinoma of the bladder with metastasis diagnosed cytologically in a pleural effusion. Acta Cytol 1999;43:1191-2.

2. Gamez RG, Jessurun J, Berger MJ, Pambuccian SE. Cytology of metastatic cervical squamous cell carcinoma in pleural fluid: report of a case confirmed by human papillomavirus typing. Diagn Cytopathol 2009;37:381-7.

3. Curry JL, Wojcik EM. Malignant pericardial effusion with metastatic squamous-cell carcinoma: discordance between ThinPrep and cell-block cytopreparation. Diagn Cytopathol 2003;29:270.

4. Hoda RS, Cangiarella J, Koss LG. Metastatic squamouscell carcinoma in pericardial effusion: report of four cases, two with cardiac tamponade. Diagn Cytopathol 1998;18:422-4.

5. Smith-Purslow MJ, Kini SR, Naylor B. Cells of squamous cell carcinoma in pleural, peritoneal and pericardial fluids. Origin and morphology. Acta Cytol 1989;33:245-53.

6. Li Q, Bavikatty N, Michael CW. The role of immunohistochemistry in distinguishing squamous cell carci- noma from mesothelioma and adenocarcinoma in pleural effusion. Semin Diagn Pathol 2006;23:15-9.

7. Pu RT, Pang Y, Michael CW. Utility of WT-1, p63, MOC31, mesothelin, and cytokeratin (K903 and CK5/ 6) immunostains in differentiating adenocarcinoma, squamous cell carcinoma, and malignant mesothelioma in effusions. Diagn Cytopathol 2008;36:20-5.

8. Graham RM, MacKinney DG, Rheault $\mathrm{MH}$ et al. The Cytologic Diagnosis of Cancer. Philadelphia; London: W. B. Saunders Company; 1954.

9. Frost JK. The Cell in Health and Disease, 2nd edn. Switzerland; Basel: Karger; 1986.

10. Kho-Duffin J, Tao LC, Cramer $\mathrm{H}$ et al. Cytologic diagnosis of malignant mesothelioma, with particular emphasis on the epithelial noncohesive cell type. Diagn Cytopathol 1999;20:57-62.

11. Sengelov L, Kamby C, von der Maase H. Pattern of metastases in relation to characteristics of primary tumor and treatment in patients with disseminated urothelial carcinoma. J Urol 1996;155:111-4.

12. Michael CW. Maligant mesothelioma. In: Serous Effusions. Davidson B, Pinar F, Michael CW. (eds). London; Dordrecht; Heidelberg; New York: Springer; 2012:pp. 79-98.

13. Ehya H. The cytologic diagnosis of mesothelioma. Semin Diagn Pathol 1986;3:196-203.

14. Stevens MW, Leong AS, Fazzalari NL, Dowling KD, Henderson DW. Cytopathology of malignant mesothelioma: a stepwise logistic regression analysis. Diagn Cytopathol 1992;8:333-41.

15. Cakir E, Demirag F, Aydin M, Unsal E. Cytopathologic differential diagnosis of malignant mesothelioma, adenocarcinoma and reactive mesothelial cells: a logistic regression analysis. Diagn Cytopathol 2009;37:4-10.

16. Xiao GQ. Cytomorphology of urothelial carcinomatous peritoneal effusion. Cytopathology 2008;19:31-3.

17. Cimino-Mathews A, Ali SZ. Metastatic urothelial carcinoma with signet ring features: cytomorphologic findings in abdominal paracentesis. Diagn Cytopathol 2011;39:132-4.

18. McGrath SM, Rana DN, Lynch M, Desai M. Metastatic transitional cell carcinoma causing a unilateral pleural effusion: a case report. Acta Cytol 2008;52:351-3.

19. Huang CC, Attele A, Michael CW. Cytomorphologic features of metastatic urothelial carcinoma in serous effusions. Diagn Cytopathol 2013;41:569-74.

20. Ordonez NG. The diagnostic utility of immunohistochemistry in distinguishing between epithelioid mesotheliomas and squamous carcinomas of the lung: a comparative study. Mod Pathol 2006;19:417-28. 